\title{
THERMOELECTRIC EFFECTS ON ELECTRICALLY CONDUCTING PARTICLES IN LIQUID METAL
}

\author{
F. Baltaretu ${ }^{1}$, J. Wang W $^{2,3}$, S. Letout ${ }^{3}$, Z.M. Ren ${ }^{2}$, \\ X. Li $i^{2}$, O.Budenkova ${ }^{3}$, Y. Fautrelle ${ }^{1}$ \\ 1 Universitatea Tehnica de Constructii Bucuresti, \\ 66 Pache Protopopescu, 0214134 Bucharest, Romania \\ ${ }^{2}$ Shanghai University, 149 Yan Chang Road, Shanghai 200072, P.R. China \\ ${ }^{3}$ SIMAP/EPM/Grenoble Institute of Technology/CNRS, \\ BP 75, 38402 Saint Martin dHres Cedex, France
}

The present paper deals with the thermoelectric effect on a solid electrically conducting particle immersed in a liquid metal under a temperature gradient. Superimposition of a static magnetic field induces thermoelectric magnetic (TEM) forces both in the solid and in the liquid. Considering a simple spherical particle, the electric current density and the corresponding forces can be calculated analytically. Two cases are considered according to the orientation of the magnetic field with respect to the temperature gradient. The corresponding flow fields around the particle are calculated in both cases. Grain motion is analyzed in the light of in-situ experiments performed earlier.

Introduction. During solidification of liquid metal, the liquid-solid interface is crossed by internal electric currents due to the Seebeck-Thomson effect when temperature differences or internal gradients exist in the material [1]. In general, all materials during solidification have a thermal gradient at the liquid-solid interface and different material properties between the liquid and the solid, including thermal and electrical conductivities, and absolute thermoelectric powers [2-8]. The existence of a temperature gradient with a non-zero component along the interface (e.g., the liquid-solid front) may cause the appearance of internal electrical current along the interface [2]. When a static magnetic field is superimposed, electromagnetic forces (TEM forces) appear both in solid and liquid phases. Many experimental evidences were obtained from post-mortem results from solidification experiments [3-8]. The electromagnetic forces acting on the solid may be responsible for grain translation, whereas fluid flows are generated in the liquid bulk around the particle. Previous in-situ solidification experiments under a vertical temperature gradient showed that equiaxed grains or dendrite fragments were deflected in a horizontal direction with respect to gravity when a transverse horizontal magnetic field was applied [7]. The present study is aimed at determining both analytically and numerically the thermoelectric forces acting on solid particles and the corresponding flow field in the vicinity of the liquid-solid interface. We consider the case of a spherical particle under a uniform static magnetic field parallel or perpendicular to the applied temperature gradient. Theoretical results allow us to interpret in-situ observations of $\mathrm{Al}-10$ wt.\% $\%$ solidification by means of X-ray imaging [8].

1. Mathematical model. In the present section, we describe the main features of the mathematical model. The geometry shown in Fig. 1 consists of a solid conducting sphere of radius $R$ or diameter $D$ in a liquid metal. It is aimed at determining the temperature field $T$ and the electric current density $\mathbf{j}$ both in the solid and in the liquid phase under steady state conditions. The basic assumptions and boundary conditions are the following: 


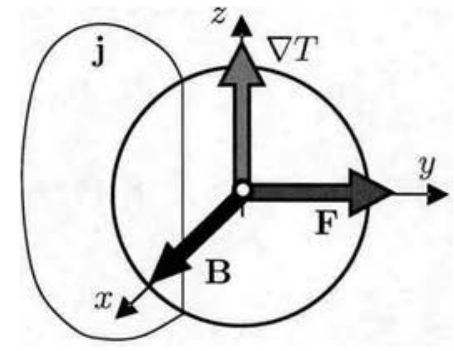

(a)

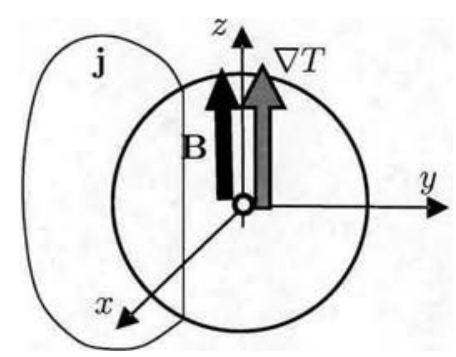

(b)

Fig. 1. Sketch of the geometry and the description of two considered cases: $(a)$ a transverse magnetic field, (b) an axial magnetic field.

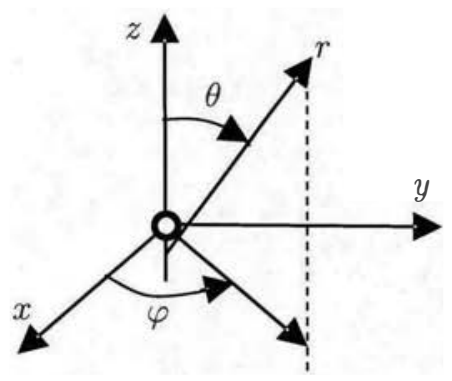

Fig. 2. Definition of the spherical polar coordinates used in the text.

- the particle velocity is zero and the fluid is at rest at infinity,

- the applied static magnetic field $\mathbf{B}$ is uniform,

- the electric current density generated by the interaction between the magnetic field and the flow field is not taken into account,

- the electric current density as well as the liquid velocities are zero at infinity,

- the temperature gradient is prescribed at infinity

$$
\nabla T=G_{\infty} \mathbf{i}_{z}
$$

with $G_{\infty}$ being a constant, and $\mathbf{i}_{z}$ is a unit vector along the $z$-axis,

- the physical properties are constant in each medium.

The coordinate systems are defined in Fig. 2.

1.1. Equations and boundary conditions. The temperature field as well as the electric current density in both media are governed by the heat equation and Ohm's law. The Ohm's law contains an additional term, which accounts for the thermoelectric effect [1]. The system is

$$
\nabla^{2} T_{\mathrm{i}}=0
$$

with $\mathrm{i}=1$ or $\mathrm{s}$, respectively, in the liquid or in the solid,

$$
j_{\mathrm{i}}=-\sigma_{\mathrm{i}} \nabla V_{\mathrm{i}}-\sigma_{\mathrm{i}} S_{\mathrm{i}} \nabla T_{\mathrm{i}} \equiv-\sigma_{\mathrm{i}} W_{\mathrm{i}},
$$

where $\sigma_{\mathrm{i}}, V_{\mathrm{i}}, S_{\mathrm{i}}$, respectively, denote the electrical conductivity, the electric scalar potential and the absolute thermoelectric power of each phases, and $W_{\mathrm{i}}=V_{\mathrm{i}}+S_{\mathrm{i}} T_{\mathrm{i}}$ 
is a generalized scalar potential. The influence of the fluid velocity $\mathbf{u}$ in the Ohm's law may be neglected as long as the Hartmann number Ha remains small.

The electric current must satisfy the continuity

$$
\nabla \cdot \mathbf{j}_{\mathrm{i}}=0 \text {. }
$$

Thus, the generalized potential is also harmonic in each phase

$$
\nabla^{2} W_{\mathrm{i}}=0 .
$$

1.2. Boundary conditions at the liquid-solid interface. These are the following (see $[1])$ :

- continuity of the normal component of the electric current density

$$
-\sigma_{\mathrm{l}} \frac{\partial V_{\mathrm{l}}}{\partial n}-\sigma_{\mathrm{l}} S_{\mathrm{l}} \boldsymbol{\nabla} T_{\mathrm{l}} \cdot \mathbf{n}=-\sigma_{\mathrm{s}} \frac{\partial V_{\mathrm{s}}}{\partial n}-\sigma_{\mathrm{s}} S_{\mathrm{s}} \boldsymbol{\nabla} T_{\mathrm{s}} \cdot \mathbf{n},
$$

with $\mathbf{n}$ being an outward unit vector normal to the sphere;

- continuity of the scalar potential

$$
V_{\mathrm{s}}=V_{\mathrm{l}}
$$

- continuity of the temperature

$$
T_{\mathrm{s}}=T_{\mathrm{l}}
$$

- continuity of the heat flux:

$$
\lambda_{\mathrm{l}} \frac{\partial T_{1}}{\partial n}=\lambda_{\mathrm{s}} \frac{\partial T_{\mathrm{s}}}{\partial n} .
$$

1.3. Boundary conditions at infinity. These are:

- the current density is zero at infinity

$$
\mathbf{j}_{1} \cdot \mathbf{n} \equiv-\sigma_{1} \frac{\partial W_{1}}{\partial n}=0
$$

- the temperature gradient is constant and oriented along the $z$-axis:

$$
\nabla T=G_{\infty} \mathbf{i}_{z}
$$

As far as the flow around the particle is concerned, the fluid is supposed to be incompressible. The velocity field is governed by the Navier-Stokes equations and mass conservation under steady-state conditions:

$$
\rho \mathbf{u} \cdot \nabla \mathbf{u}=-\nabla p+\mu \nabla^{2} \mathbf{u} \text { and } \nabla \cdot \mathbf{u}=0,
$$

with $p, \mathbf{u}, \rho, \mu$, respectively, denoting the pressure, velocity field, density and the dynamical viscosity. Fluid density variations with temperature are neglected. Thus, buoyancy forces in Eq. (12) are ignored. Orders of magnitude will be provided in Section 2 to justify the latter hypothesis. The boundary conditions are $\mathbf{u}=0$ at the particle boundary, whereas at infinity $\mathbf{u}=0$ and $p$ is a constant.

In the present case, viscous forces are usually dominant if compared with inertia. This statement is confirmed by the values of the Reynolds numbers provided in Table 2. However, for large particles, the Reynolds numbers may be of an order of one, and some inertial effects may occur as, for example, in the case of the axial magnetic field, which will be discussed later. 
1.4. Solutions for the temperature and electric current density. Because of the particular form of the boundary conditions, the solution of Eqs. (2) and (5) may be sought in spherical polar coordinates $(r, \theta, \varphi)$ in the following form (see Fig. 2 for the definition of the spherical polar coordinates):

$$
T_{\mathrm{i}}=\tilde{T}_{\mathrm{i}}(r) \cos \theta \quad \text { and } \quad W_{\mathrm{i}}=\tilde{W}_{\mathrm{i}}(r) \cos \theta .
$$

Substituting Eq. (13) into Eqs. (2) and (5) leads to general solutions, namely,

$$
T_{\mathrm{i}}=\left(\left(a_{T_{\mathrm{i}}}+\varepsilon_{\mathrm{i}} G_{\infty}\right) r+\frac{b_{T_{\mathrm{i}}}}{r^{2}}\right) \cos \theta, \quad W_{\mathrm{i}}=\left(a_{W_{\mathrm{i}}} r+\frac{b_{W_{\mathrm{i}}}}{r^{2}}\right) \cos \theta
$$

with $\varepsilon_{\mathrm{l}}=1$ and $\varepsilon_{\mathrm{s}}=0$. Using the boundary conditions, the expressions for the coefficients in Eq. (14) for both the temperature and the generalized potential are

$$
\begin{aligned}
& a_{T_{1}}=0, a_{T_{\mathrm{s}}}=G_{\infty}\left(\frac{3}{2+k}\right), b_{T_{1}}=G_{\infty} R^{3}\left(\frac{1-k}{2+k}\right), b_{T_{\mathrm{s}}}=0, a_{W_{1}}=0, \\
& a_{W_{\mathrm{s}}}=-2 \frac{\sigma_{\mathrm{l}}}{\sigma_{\mathrm{s}}} \frac{b_{W_{1}}}{R^{3}}, b_{W_{1}}=-\frac{\sigma_{\mathrm{s}}}{\sigma_{\mathrm{s}}+2 \sigma_{1}}\left(S_{\mathrm{s}}-S_{\mathrm{l}}\right)\left(\frac{3}{2+k}\right) G_{\infty} R^{3}, b_{W_{\mathrm{s}}}=0 .
\end{aligned}
$$

with $k=\lambda_{\mathrm{s}} / \lambda_{\mathrm{l}}$.

Finally, the expressions of the thermoelectric current density in both phases are

$$
\begin{gathered}
\mathbf{j}_{\mathrm{s}}=-\frac{2 \sigma_{\mathrm{s}} \sigma_{\mathrm{l}}}{\sigma_{\mathrm{s}}+2 \sigma_{1}}\left(S_{\mathrm{s}}-S_{\mathrm{l}}\right) \bar{G} \mathbf{i}_{z} \text { with } \bar{G}=\left(\frac{3}{2+k}\right) G_{\infty} \\
\mathbf{j}_{\mathrm{l}}=-\frac{\sigma_{\mathrm{s}} \sigma_{1}}{\sigma_{\mathrm{s}}+2 \sigma_{\mathrm{l}}}\left(S_{\mathrm{s}}-S_{\mathrm{l}}\right) \bar{G} \frac{R^{3}}{r^{3}}\left(2 \cos \theta \mathbf{i}_{r}+\sin \theta \mathbf{i}_{\theta}\right) .
\end{gathered}
$$

The effect of the actual temperature distribution around the particle can be taken into account by introducing a simple modified constant temperature gradient $\bar{G}$ defined in Eq. (16).

It is noticeable that the electric current density distribution in the solid is uniform, whereas in the liquid it is similar to a dipole field. Let us introduce the amplitude of the electric current density in the solid $J_{0}=\left|\mathbf{j}_{\mathbf{s}}\right|$. Under the conditions defined in Table 1, the numerical value of $J_{0}$ is $1.786 \times 10^{4} \mathrm{~A} / \mathrm{m}^{2}$.

2. Analysis of the phenomena. It is of interest to derive a characteristic velocity $U_{0}$ of the problem. It may be obtained from the balance between TEM forces and viscous ones. Indeed, the order of magnitude of the TEM force density may be obtained from Eqs. (16) and (17), namely,

$$
F_{\text {TEM }}=O\left(\frac{\sigma_{\mathrm{s}} \sigma_{\mathrm{l}}}{\sigma_{\mathrm{s}}+2 \sigma_{\mathrm{l}}}\left(S_{\mathrm{s}}-S_{\mathrm{l}}\right) \bar{G} B\right),
$$

whereas the order of magnitude $F_{\text {VIS }}$ of the viscous forces is

$$
F_{\mathrm{VIS}}=O\left(\frac{\mu U_{0}}{R^{2}}\right)
$$

Thus, Eqs. (18) and (19) yield an expression of the typical velocity scale $U_{0}$ of the problem

$$
U_{0}=\left|\frac{\sigma_{\mathrm{s}} \sigma_{\mathrm{l}}}{\sigma_{\mathrm{s}}+2 \sigma_{\mathrm{l}}}\left(S_{\mathrm{s}}-S_{\mathrm{l}}\right) \bar{G} B \frac{R^{2}}{\mu}\right| .
$$

The main non-dimensional parameters involved in the present problem are: 
- the parameter Re similar to the Reynolds number and defined from the previous estimate is as follows

$$
\operatorname{Re}=\frac{\rho_{\mathrm{l}} U_{0} R}{\mu}
$$

- the Hartmann number Ha being defined as

$$
\mathrm{Ha}=\left(\frac{\sigma_{1} B^{2} R^{2}}{\rho_{\mathrm{l}} \nu}\right)^{1 / 2} .
$$

In most cases treated in the present paper, the Hartmann number is quite weak, especially for small particles. This confirms that the electric current density derived from the $\mathbf{u} \times \mathbf{B}$ term in the Ohm's law can be neglected. Table 2 provides some numerical values of the various parameters involved in the present problem. As far as buoyancy effects are concerned, let us compare buoyancy forces to thermoelectric ones. The ratio $N$ between the two forces may be estimated by the following relation

$$
N=\rho_{\mathrm{l}} g \beta R G_{\infty}\left[\frac{\sigma_{\mathrm{s}} \sigma_{\mathrm{l}}}{\sigma_{\mathrm{s}}+2 \sigma_{\mathrm{l}}}\left(S_{\mathrm{s}}-S_{\mathrm{l}}\right) \bar{G} B\right]^{-1} .
$$

The values of $N$, which are provided in Table 2 for two particle radii, are very small. Thus, neglecting buoyancy with respect to thermoelectric forces is a reasonable approximation.

3. Determination of the electromagnetic forces and computation of the flow pattern in the axial magnetic field case. Let us consider a case, where $\mathbf{B}$ is parallel to the $z$-axis. The $\mathbf{j} \times \mathbf{B}$ electromagnetic force can be determined using Eqs. (16) and (17). Note that both the net TEM force and the net torque acting on the solid are identically zero. However, in the liquid phase the TEM force is non-zero and oriented along the azimuthal direction only. In the spherical coordinates $(r, \theta, \varphi)$ the expression of the forces in the liquid is

$$
\mathbf{F}_{\mathrm{l}}=\mathbf{j}_{1} \times B \mathbf{i}_{z}=\frac{3}{2} \frac{\sigma_{\mathrm{s}} \sigma_{\mathrm{l}}}{\sigma_{\mathrm{s}}+2 \sigma_{\mathrm{l}}}\left(S_{\mathrm{s}}-S_{\mathrm{l}}\right) \bar{G} B \frac{R^{3}}{r^{3}} \sin (2 \theta) \mathbf{i}_{\varphi} .
$$

Table 1. Numerical values of the data used in the paper for an aluminium-10 wt.\% copper alloy.

\begin{tabular}{lc}
\hline \hline & value \\
\hline Particle diameter $D,[\mu \mathrm{m}]$ & $50-300$ \\
Dynamic viscosity $\mu,[\mathrm{Pa} \cdot \mathrm{s}]$ & $1.3 \times 10^{-3}$ \\
Difference of thermoelectric constants $\Delta S=\left(S_{\mathrm{s}}-S_{\mathrm{l}}\right)[9],[\mathrm{V} / \mathrm{K}]$ & $-1.6 \times 10^{-6}$ \\
Liquid metal density $\rho,\left[\mathrm{kg} / \mathrm{m}^{3}\right]$ & 2600 \\
Magnetic field amplitude $B,[$ Tesla] & $0.008-0.08$ \\
Electrical conductivity of the liquid $\sigma_{\mathrm{l}},[\mathrm{S} / \mathrm{m}]$ & $4 \times 10^{6}$ \\
Electrical conductivity of the solid near the melting point $\sigma_{\mathrm{s}},[\mathrm{S} / \mathrm{m}]$ & $10 \times 10^{6}$ \\
Thermal conductivity of the solid $\lambda_{\mathrm{s}},[\mathrm{W} / \mathrm{m} / \mathrm{K}]$ & 150 \\
Thermal conductivity of the liquid $\lambda_{\mathrm{l}},[\mathrm{W} / \mathrm{m} / \mathrm{K}]$ & 95 \\
Thermal conductivity ratio $k$ & 1.579 \\
Coefficient of thermal expansion at liquid state $\beta,\left[\mathrm{K}^{-1}\right]$ & $1.2 \times 10^{-4}$ \\
Applied temperature gradient at infinity $G_{\infty},[\mathrm{K} / \mathrm{m}]$ & 3000 \\
Actual modified temperature gradient around the sphere $G,[\mathrm{~K} / \mathrm{m}]$ & 2514 \\
\hline \hline
\end{tabular}


Table 2. .Numerical values of some non-dimensional parameters.

\begin{tabular}{lcc}
\hline \hline Values of the parameters & \multicolumn{2}{c}{ Particle diameter } \\
for $G_{\infty}=3000 \mathrm{~K} / \mathrm{m}$ and $B=0.08 \mathrm{~T}$ & $100 \mu \mathrm{m}$ & $200 \mu \mathrm{m}$ \\
\hline Typical estimate of the velocity $U_{0}[\mathrm{~m} / \mathrm{s}]$ & $1.37 \times 10^{-3}$ & $5.50 \times 10^{-3}$ \\
Reynolds number Re & 0.137 & 1.10 \\
Hartmann number Ha & 0.222 & 0.444 \\
Ratio $N$ between buoyancy and TEM body forces & $6.42 \times 10^{-4}$ & $1.29 \times 10^{-3}$ \\
Maximum computed velocity normalized by $U_{0}$ & & \\
in the axial magnetic field case & 1.044 & 1.020 \\
Maximum computed velocity normalized by $U_{0}$ & & \\
in the transverse magnetic field case & 1.048 & 1.024 \\
\hline \hline
\end{tabular}

Note that in the case of a low Reynolds number, the Stokes equations may be solved readily analytically by assuming that the corresponding flow has a single azimuthal component $u_{\varphi}$. Presently, the fluid flow has been computed by means of the ANSYS/FLUENT commercial software in order to analyze the non-linear behaviour when the Reynolds number is of the order of unity. The geometry is three-dimensional. The latter approach allowed us to take into account the inertial effects. The expression of the body forces given by Eq. (24) was implemented in the code. The computation domain is a sphere, the radius of which is of an order of 20 times of that of a particle. This was found to be enough to minimize the confinement effect of the flow. The mesh comprises approximately 250000 nodes for small particles and 320000 for the largest ones.

In the case of a low Reynolds number, the flow pattern consists of two counterrotating azimuthal vortices in each hemisphere, as illustrated in Fig. 3. Such flow pattern is consistent with the azimuthal orientation of the thermoelectric body forces in Eq. (24). Table 2 gives some values of the computed maximum velocities

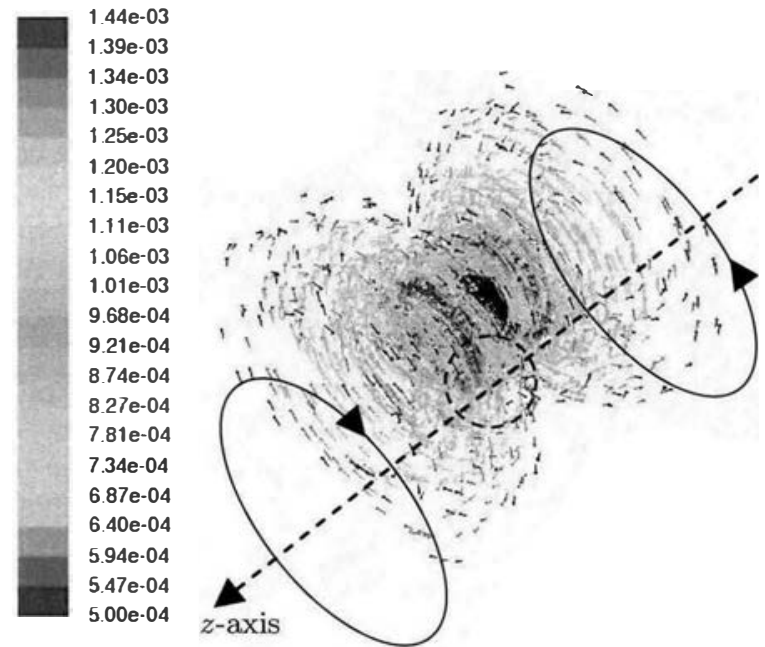

Fig. 3. View of the flow pattern around a spherical particle in the case of the axial magnetic field. The parameter values are $G_{\infty}=3000 \mathrm{~K} / \mathrm{m}$, sphere radius $R=0.05 \mathrm{~mm}$, $B=0.08 \mathrm{~T}$. The velocity scale is in $\mathrm{m} / \mathrm{s}$. General view of two counter-rotating axial vortices, $U_{\max }=1.436 \mathrm{~mm} / \mathrm{s}$. $R e=0.137$. 
(a)

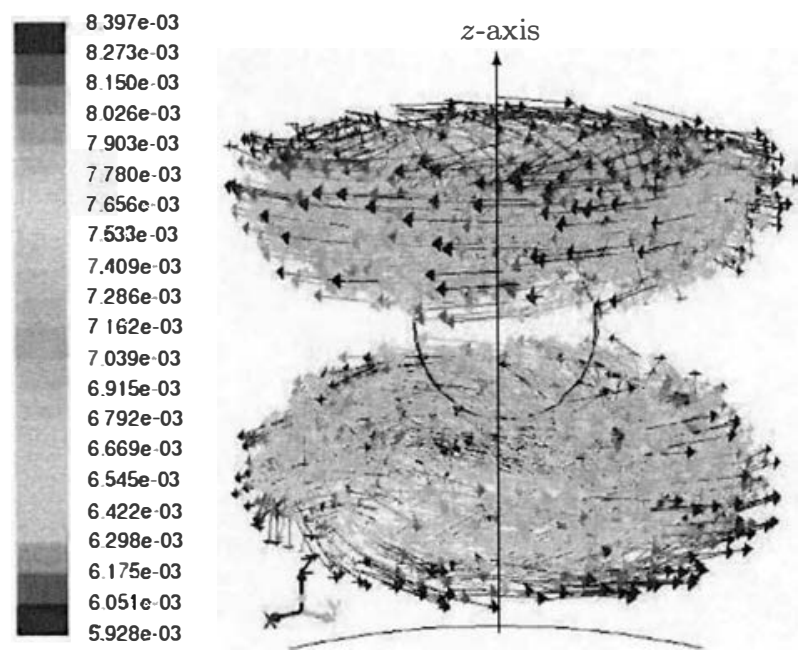

(b)

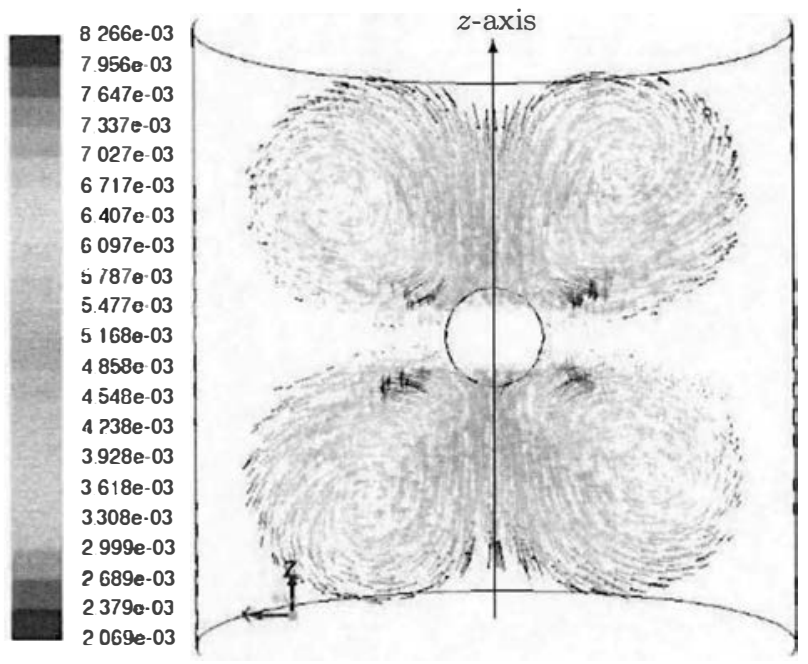

Fig. 4. View of the flow pattern around a spherical particle in the case of the axial magnetic field. The parameter values are $G_{\infty}=3000 \mathrm{~K} / \mathrm{m}$, sphere radius $R=0.15 \mathrm{~mm}$, $B=0.08 \mathrm{~T}$. The velocity scale is in $\mathrm{m} / \mathrm{s}$. (a) General view of the velocity pattern, $U_{\max }=0.0084 \mathrm{~mm} / \mathrm{s}$. (b) View of the secondary flows in the meridian plane. $R e=3.67$.

in the liquid zone. We notice that the reference velocity $U_{0}$ introduced in Eq. (20) provides a very good estimate of the TEM velocities around the sphere.

It was observed that when the Reynolds number was of the order of unity or larger, the flow pattern consisted of an azimuthal component with superimposed meridian vortices as in Taylor-Couette instability between two counter-rotating cylinders. The latter phenomenon is visible in Fig. 4 for a Reynolds number based on the reference velocity equal to 3.67. This is a consequence of the nonlinear effects due to inertial terms in the Navier-Stokes equations. Transition between a purely azimuthal pattern and a flow configuration with superimposed meridian recirculation appears when $R e$ is of the order of unity. Such behaviour is illustrated in Fig. 5, which shows the increase of the $u_{r}$ and $u_{\theta}$ components 


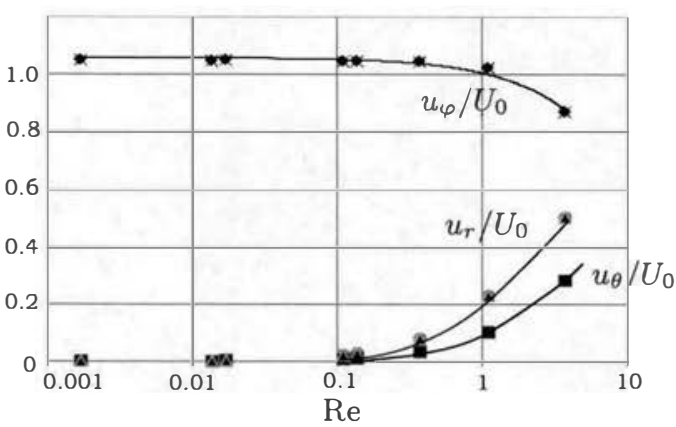

Fig. 5. Evolutions of various velocity field components vs. the Reynolds number Re. The axial magnetic field case. The temperature gradient is $G_{\infty}=3000 \mathrm{~K} / \mathrm{m}$. The magnetic field amplitude is $B=0.08 \mathrm{~T}$. The velocities are normalized by the reference velocity $U_{0}$.

when the parameter Re exceeds 0.11 approximately. We observe from Fig. 5 that the three velocity components become comparable when the Reynolds number is larger than unity. It is noticeable that the net torque acting on the sphere due to the surrounding motion is also identically zero because of the various symmetries.

\section{Determination of the electromagnetic forces and computation of} the flow pattern in the transverse magnetic field case. Let us now consider a case, where $\mathbf{B}$ is transverse and parallel to the $\boldsymbol{x}$-axis. The $\mathbf{j} \times \mathbf{B}$ electromagnetic force may be determined by using Eqs. (16) and (17). In that case, it is noteworthy that both the solid particle and the liquid one are subjected to a TEM body force. Their expressions, which may be derived from Eqs. (16) and (17), are given by Eqs. (25) and (26):

$$
\begin{aligned}
& \mathbf{F}_{\mathrm{s}}=\mathrm{j}_{\mathrm{s}} \times B \mathbf{i}_{x}=-\frac{2 \sigma_{\mathrm{s}} \sigma_{\mathrm{l}}}{\sigma_{\mathrm{s}}+2 \sigma_{\mathrm{l}}}\left(S_{\mathrm{s}}-S_{\mathrm{l}}\right) \bar{G} B \mathbf{i}_{y}, \\
& \mathbf{F}_{\mathrm{l}}=\mathbf{j}_{\mathrm{l}} \times B \mathbf{i}_{x}=-\frac{\sigma_{\mathrm{s}} \sigma_{\mathrm{l}}}{\sigma_{\mathrm{s}}+2 \sigma_{\mathrm{l}}}\left(S_{\mathrm{s}}-S_{\mathrm{l}}\right) \bar{G} B \\
& \times \frac{R^{3}}{r^{3}}\left(-\sin \theta \sin \varphi, 2 \cos \theta \sin \varphi, \cos \varphi\left(2 \cos ^{2} \theta-\sin ^{2} \theta\right)\right) .
\end{aligned}
$$

in spherical polar coordinates.

We notice that the TEM force acting on the solid is constant and oriented along the $y$-direction perpendicular both to the temperature gradient and to the magnetic field. The TEM body force is not curl-free and generates a motion of the liquid metal. The corresponding flow pattern was computed by means of ANSYS/FLUENT, and the results are illustrated in Fig. 6. The three dimensional flow pattern is quite complex because of the symmetry breaking due to the magnetic field orientation. Fig. 6 a shows that the flow configuration consists of four vortices. As in the previous case, it is noticeable that the flow configuration is sensitive to the Reynolds number.

Both pressure and viscous forces around the particle were computed. The corresponding values were much weaker than simple estimates deduced from the velocity magnitude. It seems that due to the various symmetries such net forces are identically zero. This issue is under investigation. 
(a)

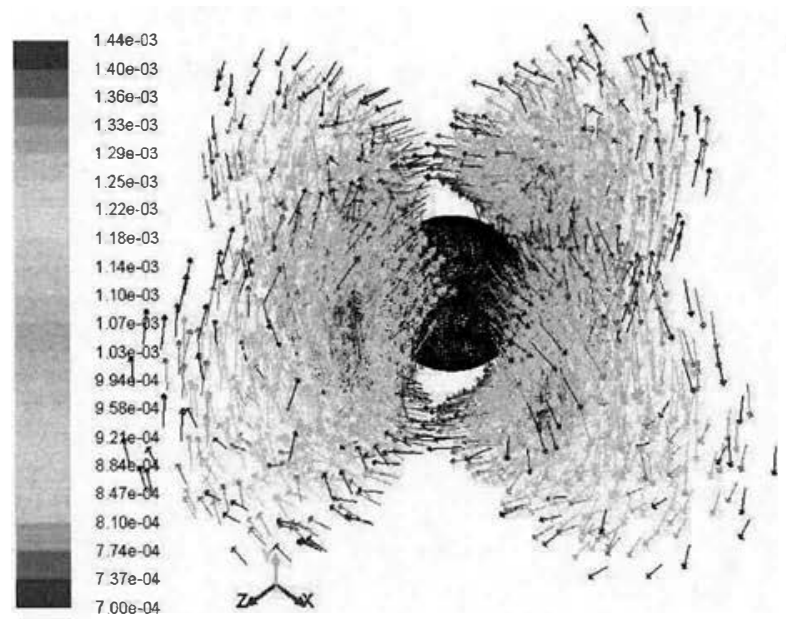

(b)
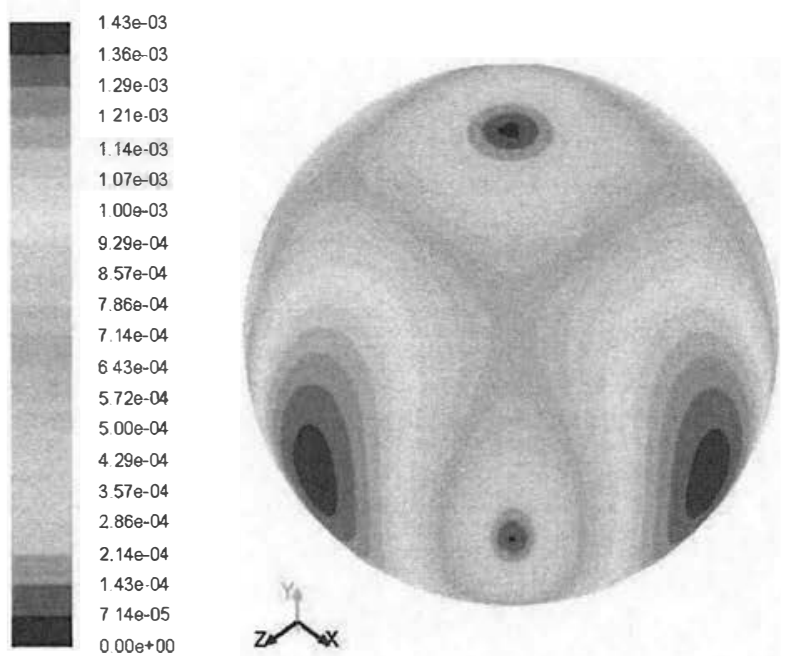

Fig. 6. TEM flow generated by a transverse magnetic field. The parameter values are $G_{\infty}=3000 \mathrm{~K} / \mathrm{m}$, sphere radius $R=0.050 \mathrm{~mm}, B=0.08 \mathrm{~T}$. The velocity scale is expressed in $\mathrm{m} / \mathrm{s}$. (a) View of the velocity vectors. (b) Distribution of the velocity amplitude on a sphere surrounding the particle, the radius of which is close to the maximum of the velocity profile, i.e., $2 R$.

5. Analysis of the confinement effects in the transverse magnetic field case. The thermoelectric current density may be modified when the particle is closely surrounded by insulating walls. In order to investigate such a phenomenon, we performed numerical modelling of the thermoelectric current around a spherical particle in a small size rectangular domain. The geometry is illustrated in Fig. 7. The particle diameters varied from $50 \mathrm{~m}$ to $190 \mathrm{~m}$, whereas the dimension of the domain was $200 \mu \mathrm{m} \times 300 \mu \mathrm{m} \times 400 \mu \mathrm{m}$. The physical parameters were identical to those used in the previous cases. In the case, where $D=190 \mathrm{~mm}$, the particle is very close to the walls. The calculations were made applying the commercial code COMSOL4.3. Two types of meshes were used. The numbers of nodes were 232537 and 555616 . We checked that the numerical results were almost identical in both cases. 


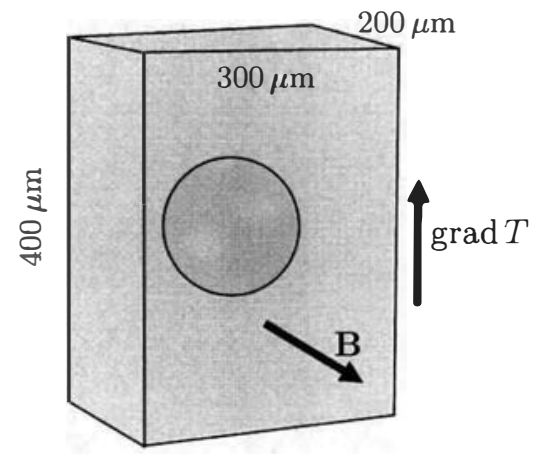

Fig. 7. Sketch of the geometry used in the computation.

Table 3. Computed values of the non-dimensional electric current density and normalized velocities for various particle radii.

\begin{tabular}{rcccc}
\hline \hline Particle diameter & $50 \mu \mathrm{m}$ & $100 \mu \mathrm{m}$ & $150 \mu \mathrm{m}$ & $190 \mu \mathrm{m}$ \\
$J / J_{0}$ & 0.996 & 0.929 & 0.781 & 0.593 \\
$U_{\max } / U_{0}$ & 0.105 & 0.085 & 0.025 & 0.013 \\
\hline \hline
\end{tabular}

The computed electric current density amplitude $J$ in the solid as well as the computed maximum liquid velocities $U_{\max }$ is listed in Table 3 . The electrical current density $J$ was normalized by $J_{0}$, which corresponds to the reference analytical solution in an infinite geometry (cf. Eq. (16)). The velocity $U_{\max }$ was normalized by $U_{0}$. It can be observed from Table 3 that the normalized electrical current density in the solid is very close to unity for $D=50 \mu \mathrm{m}$ in accordance with the analytical solution, but it decreases significantly beyond $D=100 \mu \mathrm{m}$. This may be attributed to the modification of the electrical current density due to the presence of electrically insulating walls. However, the decay of the liquid velocity around the particle is much more pronounced, even for $D=50 \mu \mathrm{m}$. It is likely that the viscous friction along the walls may be responsible for that effect.

6. Conclusions. We have demonstrated that thermoelectric-magnetic forces are able to act on electrically conducting particles immersed in a liquid metal. Thermoelectric magnetic convection may also be generated around the particle. It is noticeable that the liquid metal velocities are non-negligible even for a moderate value of the magnetic field. Both pressure and viscous forces generated by the TEM flow around the particle were found to be very weak. Under an axial static magnetic field, the net TEM force or the torque acting on the particle is identically zero. However, a liquid motion appears around the particle. When a transverse static magnetic field is applied, it is found that an electrically conducting particle is submitted to a lateral net force. That phenomenon was confirmed by in-situ $\mathrm{X}$-ray observation during solidification of liquid metals under similar conditions $[7,8]$. It was shown in $[7,8]$ that the equiaxed grains were significantly laterally deviated from the vertical direction. The deviation velocity was compatible with the simple balance between Stokes drag and the TEM force acting on the particle, although the Stokes drag underestimated the real drag force acting on the particle. The latter effect was probably caused by the finite size of the liquid domain used in the experiments as stated in [8]. 


\section{REFERENCES}

[1] J.A. Shercliff. Thermoelectric magnetohydrodynamics. J. Fluid Mechanics, vol. 91 (1979), no. 2, pp. 231-251.

[2] A.L. Gorbunov. Effect of thermoelectromagnetic convection on the production of bulk single crystals consisting of semiconductor melts in a constant magnetic field. Magnetohydrodynamics, vol. 23 (1987), no. 4, pp. 404-408.

[3] N.S. Tewari, R. Shah, H. Song. Effect of magnetic field on the microstructure and macrosegregation in directionally solidified $\mathrm{Pb}-\mathrm{Sn}$ alloys. Metall. Mater. Trans. A, vol. 25 (1994), pp. 1535-1544.

[4] Y.Y. Khine, S.J. WalkeR. Thermoelectric MHD effects during Bridgman semiconductor crystal growth with a uniform axial magnetic field. J. Cryst. Growth, vol. 183 (1998), pp. 150-158.

[5] X. Li, Y. Fautrelle, Z.M. Ren. Influence of a high magnetic field on columnar dendrite growth during directional solidification. Acta Materialia, vol. 55 (2007), pp. 5333-5347.

[6] X. Li, Y. Fautrelle, Z.M. Ren. Influence of thermoelectric effects on the solidliquid interface shape and cellular morphology in the mushy zone during the directional solidification of $\mathrm{AlCu}$ alloys under a magnetic field. Acta Mater., vol. 55 (2007), pp. 3803-3813.

[7] J. Wang, Y. Fautrelle, Z.M. Ren, X. Li, H. Nguyen-Thi, N. Mangelinck-Noel, G. Salloum Abou Jaoude, Y.B.Zhong, I. Kaldre, A. Bojarevics, L. Buligins. Thermoelectric magnetic force acting on the solid during directional solidification under a static magnetic field. Appl. Phys. Lett., vol. 101 (2012), pp. 2519041-4.

[8] G. Salloum Abou Jaoude, J. Wang, L. Abou Khalil, G. Reinhart, Z.M. Ren, N. Mangelinck-Noel, X. Li, Y. Fautrelle, H. Nguyen-Thi. Motion of equiaxed grains during directional solidification under static magnetic field. (to appear in J. Crystal Growth, 2014.)

[9] H. Yasuda, K. Inoue, Y. Minami, T. Nagira, M. Yoshiya, K. Uesugi, K. UMETANI. Direct observation of thermoelectric magnetic convection in unidirectionally solidified Al-Cu alloys. J. Iron Steel Res. Int., vol. 19S (2012), pp. 34-39. 